EPJ Web of Conferences 41, 07021 (2013)

DOI: $10.1051 /$ epjconf/20134107021

(C) Owned by the authors, published by EDP Sciences, 2013

\title{
Fluorescence Kinetics of Flavin Adenine Dinucleotide in Different Microenvironments
}

\author{
Z. Heiner ${ }^{1}$, A. Makai ${ }^{1}$, F. Sarlós ${ }^{1}$, C. Bagyinka ${ }^{1}$, A. Tóth ${ }^{1}$, G. Rákhely ${ }^{1,2}$, and G. I. Groma ${ }^{1}$ \\ ${ }^{1}$ Institute of Biophysics, Biological Research Centre, Hungarian Academy of Sciences, H-6701 \\ Szeged, Hungary \\ ${ }^{2}$ Department of Biotechnology, University of Szeged, H-6726 Szeged, Hungary
}

\begin{abstract}
Fluorescence kinetics of flavin adenine dinucleotide was measured in a wide time and spectral range in different media, affecting its intra- end extramolecular interactions, and analyzed by a new method based on compressed sensing.
\end{abstract}

\section{Introduction}

Flavin adenine dinucleotide (FAD) is a fluorescent coenzyme with substantial functions in catalyzing redox reactions. The fluorescence originates from the isoallaxazine group of the molecule, and its decaying kinetics depends highly on the interaction with the neighbouring adenine group, as well as other factors in its microenvironment $[1,2]$. This sensitivity makes FAD fluorescence kinetics an excellent tool for characterization of the metabolic state of living cells [2].

Although the fluorescence kinetics of FAD was studied extensively on different samples [1-5], the obtained data - measured on different time scales and wavelengths, and typically analyzed by simple exponential fitting procedures - are hardly comparable. $\mathrm{T}$ he aim of this work was constructing a comprehensive dataset, measured on wide time and spectral range with high time resolution in different environments, and analyzing it by advanced methods.

\section{Materials and Methods}

Fluorescence kinetics measurements were carried out on $1.5 \times 10^{-3} \mathrm{M}$ FAD dissolved in water and its 1:1 mixture with different organic solvents and on flavocytochrome (FCC) prepared from Thiocapsa roseopersicina. The samples circulating through an $1 \mathrm{~mm}$ capillary were excited at $400 \mathrm{~nm}$ by the second harmonic of a $\mathrm{T}$ i:sapphire oscillator. The fluorescence was detected by a hybrid setup, combining the technique of fluorescence up-conversion and time-correlated single photon counting, making possible to follow the kinetics up to $12 \mathrm{~ns}$ with time resolution of $\sim 250 \mathrm{fs}$ in a wide spectral range.

For the analysis of the kinetics we developed a method based on the principles of compressed sensing [6]. Our model was based on the sum of exponentials defined by a quasi-continuous set of time constants $\tau_{j}$, spanning a wide range, but it was supposed that the majority of these exponential terms have no or negligible contribution to the reconstruction of the experimental signal. We found that this problem can be optimally solved by the Basis Pursuit Denoising (BPDN) optimization procedure [7], minimizing the expression 


$$
\frac{1}{2} \sum_{i}\left(y_{i}-\sum_{j} A_{i j} x_{j}\right)^{2}+\lambda \sum_{i}\left|x_{i}\right|,
$$

where $y_{i}$ denotes the experimental data point measured at time $t_{i}, x_{j}$ represents the amplitude corresponding to the time constant $\tau_{j}, A_{i j} \equiv \exp \left(-t_{i} / \tau_{j}\right)$, and $\lambda$ is a regularization parameter.

\section{Results and Discussion}

The typical fluorescence kinetics data taken in the whole spectral range of FAD in water solution is shown in Figure 1. Since in this solvent the preferred conformational state of the molecule is a stacked one, the fluorescence is highly quenched by the adenine group, interacting via an electron transfer process, leading to a fast decay $[1,4,5]$. The corresponding distribution of the time constant values obtained by the regularized fitting according to Eq. 1 is presented in Figure 2A. The contributing time constants are aligned very well around five discrete values, almost independently of the wavelength. Note that unlike in the case of the generally used global fitting method, the BPDB procedure poses no constraint for preferring wavelength-independent time constants, hence this alignment indicates a high robustness of our approach. The fastest component around 500 fs has positive (decaying) amplitude at the low and negative (rising) one at the high end of the wavelength scale. Clearly, the underlying process can be attributed to a Stokes shift corresponding to the solvation dynamics of the neighbouring water molecules. The rest of the time constants belong to real fluorescence decaying components. This fact indicates also the robustness of the applied method, as well as the lack of distributed kinetics in this microenvironment.

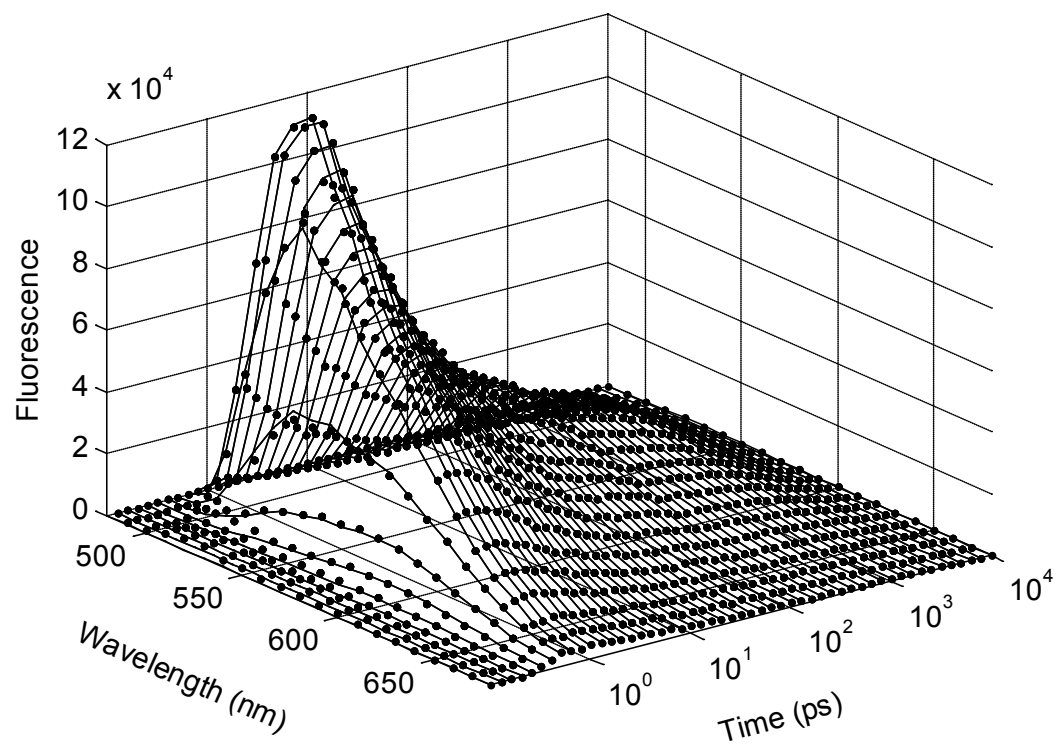

Fig.1. Experimental fluorescence kinetics of FAD in water (dots) and its fit obtained by the Basis Pursuit Denoising procedure (lines).

In the mixture of water and ethanol, dioxane or DMSO the overall decay time of the FAD fluorescence markedly increased. The results of the BPDN analysis indicate (Figure 2B) that although some shifts take place also in the positions of the contributing time constants, the main differences occur in the corresponding amplitudes. In these solvents the weight of the planar conformation of the FAD molecule is higher than in pure water, resulting in less interaction between the two groups and hence in long-lived excited state. In contrast to this, when the FAD molecule is 

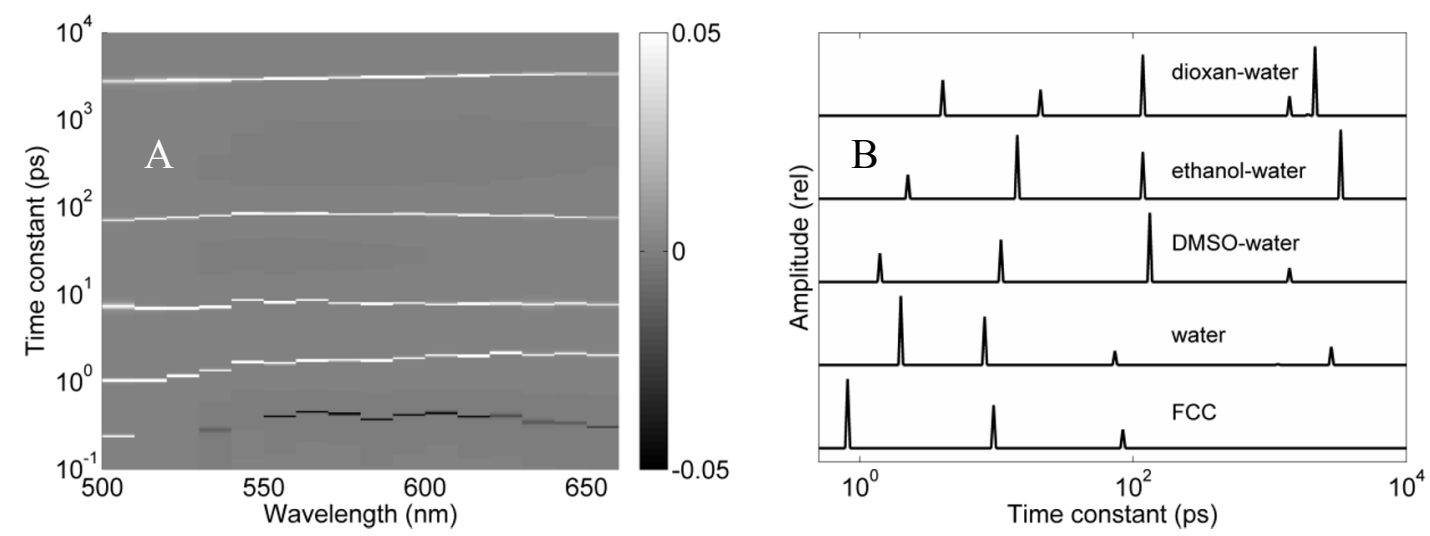

Fig.2. (A) Distribution of the amplitudes on the space of time constants and wavelengths obtained by the BPDN analysis of the kinetics shown in Figure 1. (B) Results of the BPDN analysis of FAD fluorescence kinetics in different environments at $530 \mathrm{~nm}$.

bound to FCC the slowest component attributed to the open conformation [1] is completely missing. Moreover, the smallest time constant further reduces, indicating either the existence of an even more folded conformation or additional quenching interactions with the neighbouring amino acid residues.

\section{Acknowledgement}

This work was supported by National Development Agency of Hungary (TÁMOP-4.2.1, TÁMOP4.2.2, TECH-09) and co-financed by the European Regional Fund.

\section{References}

1. T. Nakabayashi, M.S. Islam N. Ohta, J. Phys. Chem. B. 114, 15254 (2010)

2. M.C. Skala, K.M. Riching, D.K. Bird, A. Gendron-Fitzpatrick, J. Eickhoff, K.W. Eliceiri, P.J. Keely N. Ramanujam, J. Biomed. Opt. 12, 19494 (2007)

3. P.A.W. van den Berg, A. van Hoek, C.D. Walentas, R.N. Perham A. Visser, Biophys. J. 74, 2046 (1998)

4. H. Chosrowjan, S. Taniguchi, N. Mataga, F. Tanaka A.J.W.G. Visser, Chem. Phys. Lett. 378, 354 (2003)

5. L. Radoszkowicz, D. Huppert, E. Nachliel M. Gutman, J. Phys. Chem. A 114, 1017 (2010)

6. D.L. Donoho, IEEE Trans. Inf. Theory 52, 1289 (2006)

7. S.J. Kim, K. Koh, M. Lustig, S. Boyd D. Gorinevsky, IEEE J. Sel. Topics Signal Process. 1, 606 (2007) 